\title{
Impacto de la experiencia de caries en la calidad de vida relacionada a la salud bucal; Machángara, Ecuador
}

Impact of caries experience on quality of life related to bucal health, Machángara, Ecuador

Víctor Alfonso Vélez-Vásquez ${ }^{1, a}$, Ebingen Villavicencio-Caparó ${ }^{2, ~ b}$, Sandra Cevallos-Romero ${ }^{1, a}$, Cesar Del Castillo-López ${ }^{3, b}$.

\section{RESUMEN}

Objetivo: Asociar los niveles de la experiencia de caries dental con los niveles de impacto de las condiciones orales en la calidad de vida relacionada a la salud bucal. Material y métodos: Estudio descriptivo, transversal, relacional, con una muestra aleatoria de 118 escolares de la parroquia Machángara en Cuenca- Ecuador. Se empleó el índice CPOD para medir la experiencia de caries y el Child-OIDP versión Perú para medir el impacto en la calidad de vida. Fueron incluidos al estudio los escolares de 12 años que dieron su asentimiento informado y que sus padres firmaran el consentimiento informado. Se contó con el permiso del comité institucional de bioética en la carrera de Odontología de la Universidad Católica de Cuenca. Se empleó el análisis de correspondencias para asociar las variables categorizadas, también se empleó la Rho de Spearman, para la correlación de las variables en su versión de puntaje CPOD y de Child-OIDP. Resultados: No hubo correlación estadísticamente significativa entre el puntaje de experiencia de caries y su impacto en los desempeños diarios $(\mathrm{Rho}=-0.061)$, sin embargo, se encontró correspondencia entre el nivel muy alto de CPOD y el nivel muy bajo de impacto en la CVRSB y entre el nivel muy bajo de CPOD con el nivel de alto impacto en la calidad de vida relacionada a la salud bucal. Conclusiones: La asociación entre la experiencia de caries y la calidad de vida es inversa en los pacientes con altos niveles de CPOD.

PALABRAS CLAVE: Caries dental; adolescentes; calidad de vida. 


\section{SUMMARY}

Objective: To associate the levels of dental caries experience with the levels of impact of oral conditions on the quality of life related to oral health. Material and methods: A descriptive, cross-sectional, relational study with a random sample of 118 schoolchildren from the Machángara parish in Cuenca-Ecuador. The DMFT index was used to measure the experience of caries and the Child-OIDP version of Peru to measure the impact on the quality of life. The 12-year-old school children who gave their informed consent and whose parents signed the informed consent were included in the study. It was authorized by the institutional committee of bioethics in the career of Dentistry of the Catholic University of Cuenca. Correspondence analysis was used to associate the categorized variables, Spearman's Rho was also used to correlate the variables in its version of DMFT Index and Child-OIDP score. Results: There was no statistically significant correlation between the caries experience score and its impact on daily performance $(\mathrm{Rho}=-0.061)$, however there was correspondence between the very high level of DMFT and the very low level of impact on HRQoL and between the very low level of DMFT and the level of high impact on the quality of life related to oral health. Conclusions: The association between caries experience and quality of life is reversed in patients with high levels of DMFT.

\section{KEY WORDS: Dental Caries; adolescent; quality of life.}

\section{INTRODUCCIÓN}

La experiencia de caries dental es la cantidad de dientes que fueron afectados por esta enfermedad, desde la erupción de los dientes permanentes (a los 6 años aproximadamente) hasta el momento que se realiza la evaluación. Por recomendación de la Organización Mundial de la Salud (OMS)(1), se evalúa este indicador de salud a los 12 años, mediante el índice CPOD(2), que hace un conteo de las piezas cariadas actualmente, las que estuvieron cariadas y ahora están obturadas y las piezas que fueron pérdidas por caries dental. En la actualidad se considera que este valor de experiencia de caries es un fuerte predictor de la cantidad de caries que se tendrá a futuro, con una sensibilidad del $52 \%$ y una especificidad del $92 \%$ (3). Los países han adoptado este índice desde 1938 para evaluar (4) y monitorizar sus avances en la prevención de caries, de tal forma que la OMS periódicamente publica los promedios nacionales de CPOD en personas de 12 años de todos los países que coordinan sus políticas de salud con esta institución y se realizan mapas epidemiológicos con este indicador (4). Es conocido que los valores de experiencia de caries han disminuido en muchos países, de Europa y Norte América, según Kassebaum et al., ésta no ha mejorado en los últimos 20 años, a pesar de que la prevalencia de caries dental ha disminuido (5). Investigaciones en el contexto Sudamericano como el de Reinoso en 2015 indica que la parroquia rural Sayausí ciudad de Cuenca tiene un CPOD comunitario de 4,32(2,2) (6); Flores-Matamoros en 2016 reportó en la parroquia de Machángara de la misma ciudad un CPOD de 3,68(3,75) (7).

La Calidad de Vida Relacionada a la Salud Bucal (CVRSB) es un concepto de la esfera psicológica del ser humano y hace referencia a la auto percepción de las condiciones bucales y cómo estas impactan en los desempeños diarios (comer, hablar, limpiarse la boca, descansar, estado de ánimo, reír, hacer labores escolares y socializar) $(8,9)$. Uno de los primeros investigadores en plantear este concepto fue David Locker quien presenta como un concepto multidimensional debido a que el impacto puede ser en el plano funcional, social y psicológico(10). Estudios recientes en Sudamérica han reportado prevalencias del impacto de las condiciones bucales en la calidad de vida muy parecidas, como el estudio de Del Castillo en Piura-Perú que reporta 88,7\% (11); mientras que Sánchez en una población urbana de Perú reporta 82,0 (12); sin embargo ninguno de estos estudios muestra si el nivel de afectación de la salud bucal (medido con un indicador objetivo de necesidad normativa) tiene o no asociación con este gran impacto que se reporta. Precisamente el presente estudio partió de esta interrogante y planteó el objetivo de asociar el índice CPOD con la percepción del impacto de las condiciones orales en la calidad de vida relacionada a la salud bucal, y tuvo como hipótesis que a mayor cantidad de experiencia de caries mayor sería el impacto en la calidad de vida. 


\section{MATERIAL Y MÉTODOS}

Para comprobar la hipótesis planteada, se recurrió a un diseño relacional transversal bivariado.

Se estudiaron a 118 escolares de 11 y 12 años; seleccionados de manera aleatoria estratificada y proporcional en los centros educativos de la parroquia Machángara de Cuenca Ecuador el año 2017. En la muestra hubo $45 \%$ de varones y $55 \%$ de mujeres.

El encuestador fue calibrado por un experto en el manejo del instrumento Child-OIDP y por un experto en examen bucal e índice CPOD; logrando un Kappa $>0,80$. Tanto el cuestionario como el examen bucal fueron desarrollados en la mañana entre las 8 am y 10 am (antes del primer refrigerio), con luz natural y se siguieron las recomendaciones del método de encuestas bucales de la OMS para el examen bucal.

Para evaluar la CVRSB se utilizó el instrumento de Child-OIDP, validado en español y adaptado culturalmente a Perú por Bernabé et al., (13). Este cuestionario consta de 17 preguntas sobre las condiciones bucales que ha autopercibido el paciente en los últimos tres meses (dolor de muela, diente sensible, diente cariado, diente perdido, dientes separados, diente fracturado, el color del diente, forma y tamaño del diente, posición del diente, encías sangrantes, encías inflamadas, sarro dental, dolor en la boca, mal aliento, deformidad de la boca, erupción de diente permanente, pérdida de diente permanente). En caso de que el encuestado indique que percibió la condición, se le preguntaba a qué desempeño este le afectaba, luego con qué intensidad (1= leve; $2=$ moderado; ó $3=$ severo) y finalmente con qué frecuencia (de $1=$ entre una y dos veces al mes; de $2=$ entre 3 a más veces al mes y se codifica como 3 cuando sucedía 3 ó más veces por semana)(11).

Para evaluar el CPOD, se emplearon las recomendaciones de la OMS, considerando como el umbral visual de caries, la cavitación como signo de presencia de la patología (1). Para el examen intraoral se empleó una sonda de punta roma. La exploración se empezó siempre por la pieza 1.7 por oclusal, y se termió en la pieza 4.7.

Los datos fueron codificados garantizando el anonimato de los participantes y los hallazgos clínicos se registraron en un odontograma, los datos del impacto en la calidad de vida fueron registrados en el instrumento documental del CHILD-OIDP y luego llevados a la computadora mediante el programa de acceso libre Epi. Info ver. 7.1; con el cual se grabaron los datos y se realizó un control de calidad del registro de datos, luego se exportaron a un archivo de Excel, en el que se realizó la depuración de los datos, cálculo de variables de interés.

Finalmente los datos se trasladaron al programa SPSS ver.17 para el análisis estadístico, el cual incluyó el análisis descriptivo univariado, el cálculo de la prueba de Chi cuadrado, para asociar los niveles de CPOD con la prevalencia de impacto; la $\mathrm{R}$ de Pearson para relacionar el puntaje de CPOD con el puntaje de Impacto en la calidad de vida y el análisis de correspondencia simple para evaluar la correlación entre estas mismas variables.

El estudio fue inscrito y aprobado por el comité de investigación de la carrera de Odontología de la Universidad Católica de Cuenca Ecuador con número registro 017-2016; al igual que se dio aprobación al consentimiento informado y al asentimiento, los cuales fueron firmados por los examinados y sus padres. Se empleó un código alfanumérico y clave de ingreso a la base de datos de único conocimiento del investigador principal para garantizar el anonimato. Esta investigación es un análisis adicional que parte de la tesis de grado presentada en la Universidad Católica de Cuenca- Ecuador, defendida por el autor principal del presente artículo.

\section{RESULTADOS}

Se evaluó una muestra de 118 escolares entre 11 y 12 años, matriculados en las instituciones educativas de la parroquia Machángara.

Respecto a la variable impacto en los desempeños diarios, el $88,1 \%$ indican tener al menos un desempeño impactado, los desempeños con mayor prevalencia de impacto fueron comer alimento y realizar la limpieza de los dientes con $80,5 \%$ y $69,5 \%$ respectivamente (Tabla 1). En cuanto a la variable condiciones que causan impacto en la salud bucal, encontramos que las condiciones más frecuentes fueron diente sensible en el $46,6 \%$ de los pacientes, de igual forma el dolor de muela y mal aliento se presentaron en el 32,2\%. Al comparar las condiciones 
Tabla 1. Análisis del impacto en los desempeños diarios

\begin{tabular}{|c|c|c|c|c|c|c|c|c|c|}
\hline & $\begin{array}{c}\text { Comer } \\
\text { alimentos }\end{array}$ & $\begin{array}{l}\text { Hablar } \\
\text { claro }\end{array}$ & Limpieza & Descanso & $\begin{array}{c}\text { Estado de } \\
\text { animo }\end{array}$ & $\begin{array}{l}\text { Sonreir y } \\
\text { reir }\end{array}$ & Labores & Socializar & $\begin{array}{c}\text { Todas las } \\
\text { actividades } \\
\text { diarias }\end{array}$ \\
\hline $\mathrm{n}$ & 95 & 59 & 82 & 46 & 71 & 71 & 50 & 52 & 104 \\
\hline$\%$ & 80,5 & 50,0 & 69,5 & 39,0 & 60,2 & 60,2 & 42,4 & 44,1 & 88,1 \\
\hline \multicolumn{10}{|c|}{ Puntaje de impactos Child-OIDP } \\
\hline Media(d.e.) & $3,38(3,08)$ & $2,28(3,09)$ & $2,97(3,10)$ & $1,66(2,88)$ & $2,51(3,11)$ & $2,89(3,27)$ & $2,30(3,36)$ & $2,09(3,11)$ & $20,08(20,87)$ \\
\hline $\begin{array}{l}\text { Rango } \\
\text { min-máx }\end{array}$ & $0-9$ & $0-9$ & $0-9$ & $0-9$ & $0-9$ & $0-9$ & $0-9$ & $0-9$ & $0-72$ \\
\hline $\begin{array}{l}\text { Percentiles } \\
(25,50,75)\end{array}$ & $(1 ; 2 ; 6)$ & $(0 ; 0,5 ; 4,0)$ & $(0 ; 2,0 ; 6,0)$ & $(0 ; 0 ; 2)$ & $(0 ; 1,0 ; 4,0)$ & $(0 ; 2,0 ; 6,0)$ & $(0 ; 0 ; 4,0)$ & $(0 ; 0 ; 4,0)$ & $(3,0 ; 13,0 ; 30,0)$ \\
\hline \multicolumn{10}{|c|}{ Intensidad de impactos ( $\%$ de escolares con impactos $) \mathrm{n}=118$} \\
\hline $\begin{array}{l}\text { Sin } \\
\text { impacto }\end{array}$ & 19,5 & 50,0 & 30,5 & 61,0 & 39,8 & 40,7 & 58,7 & 57,6 & 11,9 \\
\hline Muy poco & 14,4 & 8,5 & 12,7 & 10,2 & 11,0 & 8,5 & 5,1 & 5,9 & 30,5 \\
\hline Poco & 21,2 & 9,3 & 16,9 & 8,5 & 18,6 & 11,0 & 5,1 & 5,9 & 15,3 \\
\hline Regular & 16,1 & 11,9 & 13,6 & 5,9 & 9,3 & 12,7 & 9,3 & 11,9 & 21,2 \\
\hline Severo & 12,7 & 8,5 & 12,7 & 4,2 & 7,6 & 11,9 & 6,8 & 6,8 & 7,6 \\
\hline $\begin{array}{l}\text { Muy } \\
\text { severo }\end{array}$ & 16,1 & 11,9 & 13,6 & 10,2 & 13,6 & 15,3 & 15,3 & 11,9 & 13,6 \\
\hline
\end{tabular}

(D.E.) Desviación estándar

de acuerdo con el sexo, solo se encontró diferencia estadísticamente significativa en llagas o heridas en la boca, siendo más frecuente en las mujeres $(p=0,01)$ y erupción de dientes permanentes, con ventaja en las mujeres ( $p=0,013)$ (Tabla 2). Al comparar el impacto en cada desempeño de acuerdo a sexo, no se encontró diferencia significativa en ningún caso (tabla 3 ). En promedio los pacientes estudiados tienen 3,65 desempeños afectados, al comparar este promedio mediante la prueba U de Mann Withney entre varones y mujeres no se encontró diferencia estadísticamente significativa (tabla 4). Al evaluar el puntaje de impacto en cada desempeño se encontró que el más afectado fue el de comer alimentos, seguido por el de limpiarse los dientes. A pesar de que el impacto en el desempeño dormir no es uno de los más frecuentes, si se encontró diferencia estadísticamente significativa entre varones y mujeres $\mathrm{p}=0,026$; comprobado mediante la prueba U de Mann Withney (tabla 5).

En cuanto a la variable caries, la prevalencia fue de $83,1 \%$ y no se encontró diferencia estadísticamente significativa entre varones y mujeres. El promedio de CPOD en la muestra total fue de 3,89 (D.E. 3,45) lo cual indica que hay una gran variación de los datos y a pesar de que las mujeres presentan un promedio más elevado esta diferencia no fue estadísticamente significativa al valor encontrado en varones ( $U$ de
Mann Withney). De igual forma la prevalencia de caries activas en la muestra total es elevada $(83,1 \%)$ y a pesar de que existe una diferencia de $9 \%$ de prevalencia entre varones y mujeres está no es estadísticamente significativa (Chi cuadrado). Respecto a los niveles de caries, el 38\% de los pacientes estudiados tienen niveles entre Alto y Muy alto. De igual forma no hubo diferencia al comparar la distribución de frecuencias de los niveles de experiencia de caries entre varones y mujeres mediante la prueba estadística Chi cuadrado (tabla 6).

Para asociar las dos variables se siguieron dos estrategias de análisis, la primera fue el análisis de correspondencias, en la cual se utilizaron los datos cualitativos ordinales de ambas variables, el mencionado análisis demostró una cohesión de los datos en tres grupos, donde se formaron tres grupos de datos correspondientes, en el primer grupo se juntaron los casos de nivel bajo de CPOD con los de nivel severo del impacto; de igual forma se juntaron los casos que presentaron muy alto nivel de CPOD con los que tiene un nivel de muy poco impacto en la calidad de vida, finalmente formaron un tercer grupo los casos de nivel medio o de muy bajo nivel de CPOD con los que presentaron nivel regular o poco nivel de severidad de impacto (gráfico 1). 
Tabla 2. Análisis de las condiciones como causa de impacto.

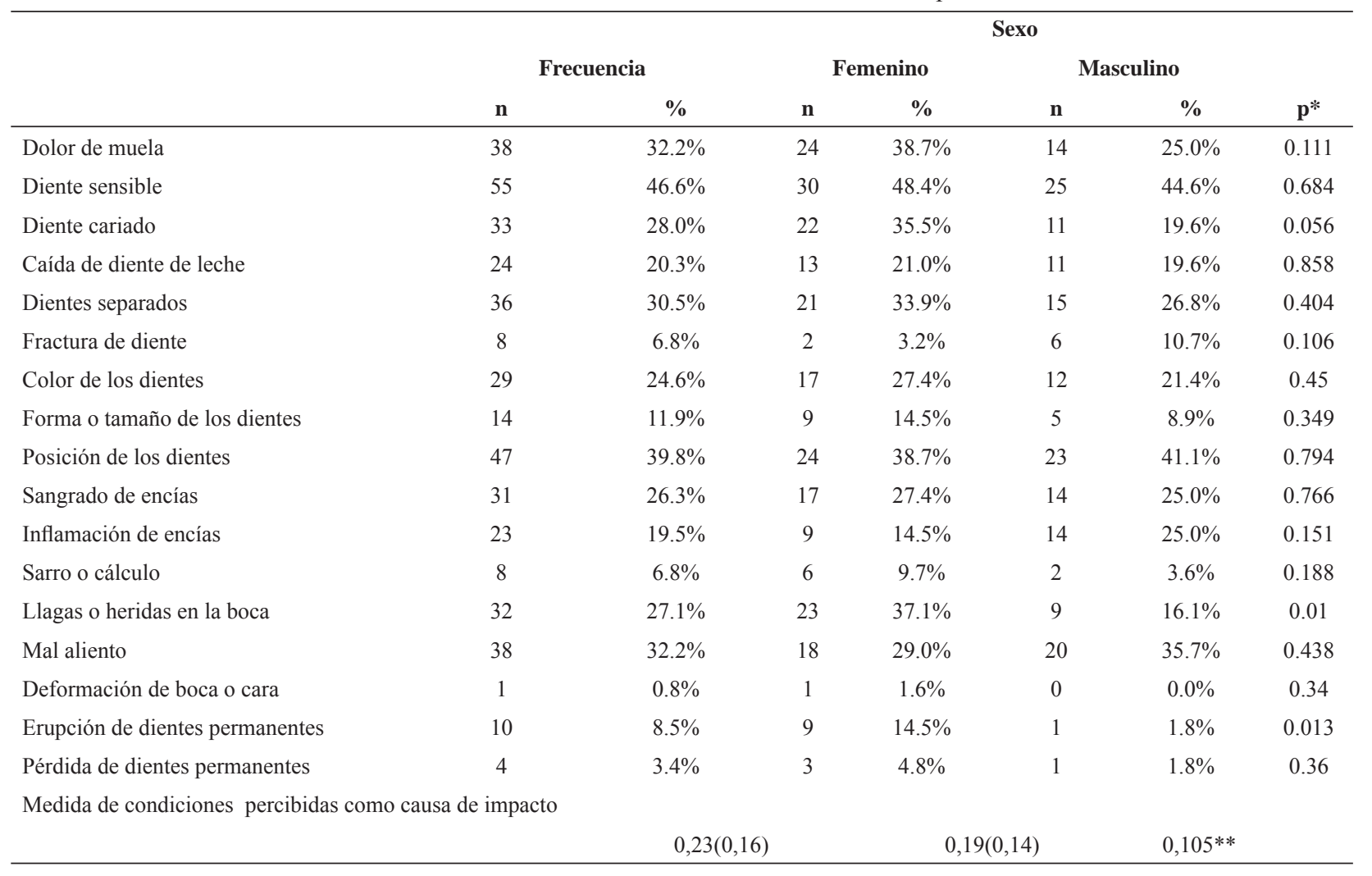

Media (d.e.)
$*$ Chi cuadrado $* *$ U de Man Withney

Tabla 3. Análisis del impacto en cada desempeño de acuerdo al sexo.

\begin{tabular}{|c|c|c|c|c|c|c|}
\hline & \multicolumn{6}{|c|}{ Sexo } \\
\hline & \multirow[b]{2}{*}{ Impacto } & \multicolumn{2}{|c|}{ Femenino } & \multicolumn{2}{|c|}{ Masculino } & \multirow[b]{2}{*}{ Sig. } \\
\hline & & $\mathrm{N}$ & $\%$ & $\mathbf{N}$ & $\%$ & \\
\hline \multirow{2}{*}{ Comer alimentos } & NO & 10 & $16,1 \%$ & 13 & $23,2 \%$ & \multirow{2}{*}{0,332} \\
\hline & SI & 52 & $83,9 \%$ & 43 & $76,8 \%$ & \\
\hline \multirow{2}{*}{ Hablar claro } & NO & 30 & $48,4 \%$ & 29 & $51,8 \%$ & \multirow{2}{*}{0,712} \\
\hline & SI & 32 & $51,6 \%$ & 27 & $48,2 \%$ & \\
\hline \multirow{2}{*}{ Limpieza de dientes } & NO & 17 & $27,4 \%$ & 19 & $33,9 \%$ & \multirow{2}{*}{0,443} \\
\hline & SI & 45 & $72,6 \%$ & 37 & $66,1 \%$ & \\
\hline \multirow{2}{*}{ Descanso } & NO & 33 & $53,2 \%$ & 39 & $69,6 \%$ & \multirow{2}{*}{0,068} \\
\hline & SI & 29 & $46,8 \%$ & 17 & $30,4 \%$ & \\
\hline \multirow{2}{*}{ Estado de animo } & NO & 21 & $33,9 \%$ & 26 & $46,4 \%$ & \multirow{2}{*}{0,164} \\
\hline & SI & 41 & $66,1 \%$ & 30 & $53,6 \%$ & \\
\hline \multirow{2}{*}{ Sonreir y reir } & NO & 20 & $32,3 \%$ & 27 & $48,2 \%$ & \multirow{2}{*}{0,077} \\
\hline & SI & 42 & $67,7 \%$ & 29 & $51,8 \%$ & \\
\hline \multirow{2}{*}{ Labores } & NO & 35 & $56,5 \%$ & 33 & $58,9 \%$ & \multirow{2}{*}{0,786} \\
\hline & SI & 27 & $43,5 \%$ & 23 & $41,1 \%$ & \\
\hline \multirow{2}{*}{ Socializar } & NO & 32 & $51,6 \%$ & 34 & $60,7 \%$ & \multirow{2}{*}{0,320} \\
\hline & SI & 30 & $48,4 \%$ & 22 & $39,3 \%$ & \\
\hline \multirow{2}{*}{ Almenos uno } & NO & 6 & $9,7 \%$ & 8 & $14,3 \%$ & \multirow{2}{*}{0,440} \\
\hline & SI & 56 & $90,3 \%$ & 48 & $85,7 \%$ & \\
\hline
\end{tabular}


Tabla 4. Extensión del impacto de las condiciones bucales sobre los desempeños

\begin{tabular}{lcccccc}
\hline & n & Media & Desv. Estándar & Mínimo & Máximo & p \\
\hline Total & 118 & 3.65 & 2.56 & 0 & 11 & $0,105^{*}$ \\
Mujeres & 62 & 4.00 & 2.65 & 0 & 11 & \\
Varones & 56 & 3.27 & 2.42 & 0 & 10 & \\
\hline & & & & Prueba U de Mann Withney
\end{tabular}

\begin{tabular}{|c|c|c|c|c|c|c|}
\hline & \multirow{3}{*}{$\begin{array}{c}\text { TOTAL } \\
\text { Media (D.E) }\end{array}$} & \multicolumn{4}{|c|}{ SEXO } & \multirow{3}{*}{ SIG } \\
\hline & & \multicolumn{2}{|c|}{ FEMENINO } & \multicolumn{2}{|c|}{ MASCULINO } & \\
\hline & & Media & (D.E.) & Media & (D.E.) & \\
\hline COMER ALIMENTOS & $3,38(3,08)$ & 4,0 & 3,0 & 3,0 & 3,0 & N.A. \\
\hline HABLAR CLARO & $2,28(3,09)$ & 2,7 & 3,4 & 1,8 & 2,6 & 0,292 \\
\hline LIMPIEZA & $2,97(3,10)$ & 3,3 & 3,3 & 2,6 & 2,8 & 0,253 \\
\hline DESCANSO & $1,66(2,88)$ & 2,3 & 3,4 & 0.9 & 1,9 & 0,026 \\
\hline ESTADO DE ANIMO & $2,51(3,11)$ & 3,0 & 3,4 & 1,9 & 2,7 & 0,107 \\
\hline SONREIR Y REIR & $2,89(3,27)$ & 3,4 & 3,6 & 2,3 & 2,9 & 0,067 \\
\hline LABORES & $2,30(3,36)$ & 2,4 & 3,5 & 2,1 & 3,2 & 0,627 \\
\hline SOCIALIZAR & $2,09(3,11)$ & 2,3 & 3,2 & 1,8 & 3,0 & 0,416 \\
\hline $\begin{array}{l}\text { TODAS LAS } \\
\text { ACTIVIDADES DIARIAS }\end{array}$ & $20,08(20,87)$ & 23,4 & 23,7 & 16,5 & 16,7 & 0,183 \\
\hline
\end{tabular}

Tabla 6. Aanálisis de CPOD de acuerdo con el sexo

\begin{tabular}{|c|c|c|c|c|}
\hline & TOTAL & FEMENINO & MASCULINO & SIG. \\
\hline \multicolumn{5}{|c|}{ Estadísticos de CPOD } \\
\hline $\mathrm{n}$ & 118 & 62 & 56 & \multirow{5}{*}{$0,534^{*}$} \\
\hline Media & 3.59 & 4.05 & 3.71 & \\
\hline D.E. & 3.45 & 3.47 & 3.44 & \\
\hline Mínimo & 0 & 0 & 0 & \\
\hline Máximo & 14 & 14 & 14 & \\
\hline \multicolumn{5}{|c|}{ Prevalencia de caries } \\
\hline & $\mathrm{n}(\%)$ & $\mathrm{n}(\%)$ & $\mathrm{n}(\%)$ & \\
\hline ENFERMOS & $98(83,1)$ & $54(87,1)$ & $44(78,6)$ & \multirow{2}{*}{$0,218^{* *}$} \\
\hline SANOS & $20(16,9)$ & $8(12,9)$ & $12(21,4)$ & \\
\hline \multicolumn{5}{|c|}{ Niveles de CPOD } \\
\hline MUY ALTO & $23(19,5)$ & $12(19,4)$ & $11(19,6)$ & \multirow{6}{*}{$0,969 * *$} \\
\hline ALTO & $22(18,6)$ & $13(21,0)$ & $9(16,1)$ & \\
\hline MEDIO & $22(18,6)$ & $11(17,7)$ & $11(19,6)$ & \\
\hline BAJO & $13(11,0)$ & $7(11,3)$ & $6(10,7)$ & \\
\hline MUY BAJO & $38(32,2)$ & $19(30,6)$ & $19(33,9)$ & \\
\hline Total & $118(100)$ & $62(100,0)$ & $56(100,0)$ & \\
\hline
\end{tabular}




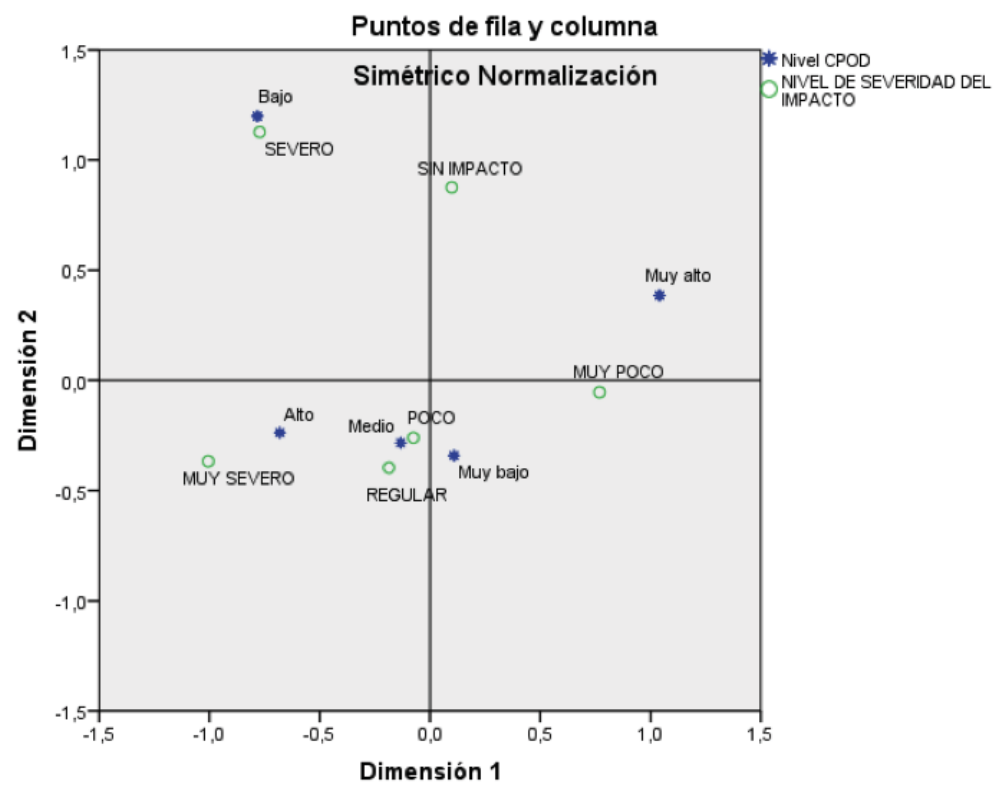

Gráfico 1. Análisis de correspondencias entre el nivel de cpod y nivel de impacto de las condiciones orales en la calidad de vida relacionada a la salud bucal.

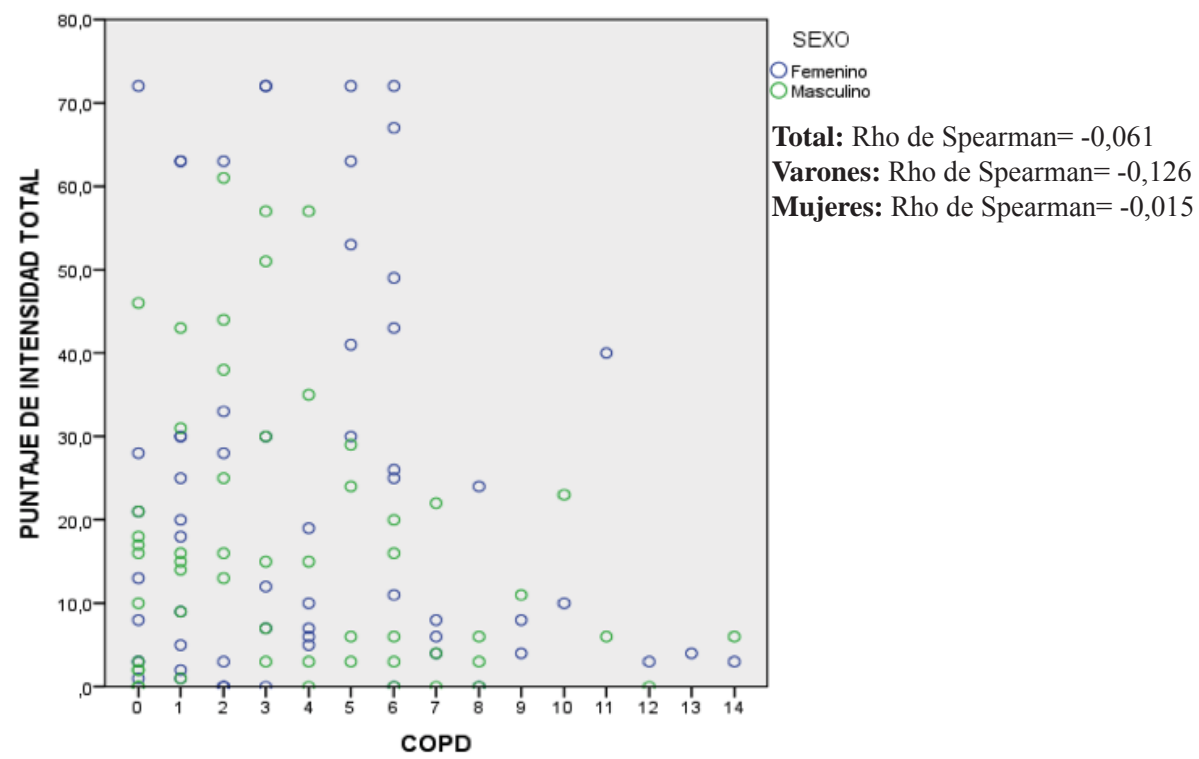

Gráfico 2. Correlación entre el puntaje de CPOD y el puntaje de intensidad de impacto de las condiciones en la calidad de vida.

Dado que se presentó una inconsistencia en la asociación esperada entre los niveles de CPOD y los niveles de impacto en la CVRSB, se decidió analizar este mismo cotejo de variables mediante la segunda estrategia empleando la correlación de Rho de Spearman $(\mathrm{Rho}=-0,061)$, en un diagrama de dispersión, el cual nos corrobora que los casos con mayor puntaje de CPOD, a la vez tienen puntajes muy bajos de impacto en la CVRSB, como es el caso de los dos pacientes que tienen valores de 14 en el CPOD, en quienes se corrobora que sus puntajes de impacto no llegan ni a 10 puntos; por el contrario los pacientes con muy bajos puntajes de CPOD ( de 1 a 3) muestran los valores más altos de impacto en 
su CVRSB (valores superiores a 60 puntos), llama la atención que los pacientes entre 7 y 10 puntos, en cuanto a impacto no llegan ni a 30 puntos (gráfico 2). Estos hallazgos corroboran las tendencias mostradas en el análisis de correspondencias (gráfico 1). El valor negativo del estadístico Rho, indica la relación inversa entre ambas variables.

\section{DISCUSIÓN}

El valor de CPOD comunitario encontrado en esta población fue de 3,89 (D.E. 3,45) tiene semejanza con lo reportado en Ecuador por Reinoso que reportó 4,32 (D.E. 2,2) a la misma edad (6). Sin embargo este resultado no coincide con lo encontrado en Valle Noguén Concepción Chile por Contreras que reporta 6,8 (D.E. 3,42 ) probablemente esta diferencia se deba a que el presente estudio fue realizado en una zona urbana de clase media y el estudio de Chile se realizó en una población rural turística de su provincia (14). Otro estudio en el que se evaluó esta asociación entre caries dental y calidad de vida es el publicado por Vijay (15) con quien tampoco coincide el valor promedio de CPOD, dado que este reporta 2,11 (D.E. 2,05 ) en varones y 2,61 ( D.E. 2,52 ) en mujeres de 12 años en el distrito Ernakulam de Kerala en India, zona de mayor desarrollo comercial y financiero de su estado, esta diferencia también es explicable debido a la diferente gradiente de ingresos económicos entre ambos lugares. Otro estudio realizado en Colombia por Ramirez-Puerta reportó 1,6 (D.E. 1,7) de CPOD comunitario (16), lo que tampoco coincide con los datos hallados en el presente estudio, probablemente debido a la larga tradición de prevención y promoción de la salud que tiene el sistema de salud Colombiano. En cuanto al impacto en la calidad de vida, los resultados de este estudio muestran que los participantes perciben el impacto de las condiciones bucales sobre su calidad de vida relacionada a la salud bucal en un $88,1 \%$; dato que coincide con lo reportado por Del Castillo et al., en la ciudad de Piura Perú que encontraron $88,7 \%$ de escolares que tuvieron al menos un desempeño diario impactado (11). También Verdugo-Encalada encontró valores similares $(75,5 \%)$ en una población urbano rural del cantón Cañar en el Ecuador (17). Sin embargo estos datos no coinciden con lo hallado por ReinosoVintimilla en Sayausí Ecuador que reporta un 98,9\% de prevalencia de impacto (9), debido a que se estudia en una población rural a diferencia del presente estudio que se realizó en zona urbana. Los hallazgos del presente estudio tampoco coinciden con lo hallado por Paredes-Martinez en un distrito de clase media baja de la ciudad de Lima Perú quien reporta un 100\% de percepción del impacto de la condiciones orales sobre la calidad de vida relacionada a la salud bucal, otro estudio con el que no se coincide es el realizado por Ingunza en Perú, reporta un $100 \%$ de prevalencia de impacto (18), a diferencia del presente estudio que encuentra $12 \%$ menos de impacto, probablemente esta diferencia se deba a que el estudio en Perú se realizó en una zona urbano marginal, donde las condiciones económicas son precarias y el acceso a servicios de salud bucal presenta una barrera financiera muy fuerte.

El análisis de asociación entre los niveles de CPOD y los niveles de impacto en la calidad de vida mostraron que hay una asociación inversa entre estas dos variables, por lo que los pacientes con mayor nivel de caries tienen menor impacto en sus actividades diarias y los pacientes con menores índices de CPOD manifestaron los niveles mayores de impacto, lo que se puede explicar debido a que quienes tienen mayor cantidad de piezas cariadas, o son resistentes al dolor y malestares ocasionados por una mala salud bucal o simplemente se adaptaron y por ese motivo no están motivados a mejorar su condición de salud bucal (gráfico 1). En el lado opuesto están los pacientes que tienen mejor salud bucal y que al tener alguna condición que perciben, les genera impacto en su calidad de vida, por lo que es esperable que busquen atención dental y esta motivación podría frenar el avance del proceso de deterioro de la salud bucal.

La información acerca de la asociación entre la experiencia de caries y el impacto en la calidad de vida relacionada a la salud bucal es importante porque abre una nueva visión de cómo resolver el problema de la alta prevalencia de las enfermedades bucales, dado que por mucho tiempo se ha pensado que a mayor cantidad de piezas afectadas, debería ser mayor el impacto, sin embargo la presente investigación ha puesto de manifiesto que esto no es así, muy por el contrario parece que las personas con mayor cantidad de piezas afectadas, tienen menor impacto en sus desempeños diarios, por algún mecanismo de adaptación, lo que coincide con lo planteado por Adunola denominando a este fenómeno la paradoja de la necesidad dental, concepto que consiste en que aquellos que no reciben atención dental son lo que 
más necesidad presentan (19).

Una limitación del presente estudio es la estratificación en niveles socio económicos y de los niveles de instrucción de los padres o cuidadores de estos escolares, que son dos puntos fundamentales para entender por completo el acceso a servicios de salud bucal y lograr un esquema de patrón de comportamiento, que se pueda modificar en función de hacer una prevención y promoción de la salud más científica, sustentada por la teoría del cambio de conducta.

\section{CONCLUSIONES}

Los hallazgos de este estudio sugieren que la calidad de vida recibe el impacto de las condiciones de salud bucal, en una forma diferente a la que se tenía pensada hasta el momento. A partir de 7 puntos de CPOD el impacto disminuye convirtiéndose en casi nulo en valores altos del mismo. Es necesario hacer nuevos estudios que indaguen acerca del punto de corte exacto en el que la calidad de vida ya no se impacta más a medida que se deteriora aún más la salud bucal. Este punto de corte es el límite para encontrar a un paciente que se motive en la mejora de su salud bucal.

Todos los autores declaran no tener conflicto de intereses.

Aprobación del comité de investigación de la carrera de Odontología de la Universidad Católica de Cuenca Ecuador con número registro 017-2016.

El presente estudio fue autofinanciado.

\section{Correspondencia:}

Cesar Del Castillo-López

Correo electrónico: cesar.del.castillo.1@upch.pe

\section{REFERENCIAS BIBLIOGRÁFICAS}

1. World Health Organization. Oral health surveys: basic methods. Sao Paulo: World Health Organization; 2013.

2. Radić M, Benjak T, Vukres VD, Rotim Ž, Zore IF. Presentation of DMFT/dmft Index in Croatia and Europe. Acta Stomatol Croat. 2015;49(4):275-84.

3. Motohashi M, Yamada H, Genkai F, et al. Employing $\mathrm{dmft}$ score as a risk predictor for caries development in the permanent teeth in Japanese primary school girls. J Oral Sci. 2006;48(4):233-7.
4. Petersen PE, Bourgeois D, Ogawa H, Estupinan-Day S, Ndiaye C. Policy and Practice The global burden of oral diseases and risks to oral health. Bulletin of the World Health Organization. 2005; 83:0-0.

5. Kassebaum NJ, Smith AGC, Bernabé E, et al. Global, regional, and national prevalence, incidence, and disability-adjusted life years for oral conditions for 195 Countries, 1990-2015: A systematic analysis for the global burden of diseases, injuries, and risk factors. J Dent Res. 2017;96(4):380-7.

6. Reinoso N, Villavicencio E. Caries dental en escolares de 12 años de la parroquia Aayausí (Cuenca) Ecuador. Rev Odontol Act. 2016;1(1):32-7.

7. Flores-Matamoros L, Reinoso-Vintimilla NR, TelloLarriva M, Abril-Pesantez J. Epidemiología de la caries en niños de 12 años en Machángara, Ecuador. Evidencias en Odontol Clínica. 2018;3(2):34-9. (Citado el 20 de enero de 2019) Disponible en: https:// revistas.uancv.edu.pe/index.php/EOC/article/view/493

8. Chaffee BW, Rodrigues PH, Kramer PF, Vítolo MR, Feldens CA. Oral health-related quality of life measures: variation by socioeconomic status and caries experience. Community Dent Oral Eidemiol. 2017; 45(3):216-24. (Citado el 20 de enero de 2019) Disponible en: https:// www.ncbi.nlm.nih.gov/pmc/articles/PMC5506781/ pdf/nihms872547.pdf

9. Reinoso-Vintimilla N, Del Castillo-López C. Calidad de vida relacionada a la salud bucal en escolares de Sayausí, Cuenca Ecuador. Rev Estomatológica Hered.2017; 27(4): 227. DOI: https://doi.org/10.20453/ reh.v27i4.3214

10. Slade GD. Measuring oral health and quality of life. Chapel Hill: Department of Dental Ecology,University of North Carolina; 1997.

11. Del Castillo-López C, Evangelista-Alva A, SánchezBorjas P. Impacto de la condiciones bucales sobre la calidad de vida en escolares de ámbito rural, Piura, Perú. Rev Estomatológica Hered. 2014;24(4):220-228 . DOI: https://doi.org/10.20453/reh.v24i4.2164

12. Sánchez-Borjas P. Impacto de las condiciones orales sobre la calidad de vida en escolares de 11 y 12 años de edad de Zapallal-Puente Piedra. Odontol Act Rev Científica. 2017;2(2):7-16.

13. Bernabé E, Sheiham A, Tsakos G. A comprehensive evaluation of the validity of Child-OIDP: further evidence from Peru. Community Dent Oral Epidemiol. 2008; 36(4):317-25.

14. Contreras I, Contreras E, Araneda P, Solis B. Oral health status in 12 to 17 -year-old school children from Valle Nonguén, Concepción, 2013. J Oral Res. 2013;2(3):109-13.

15. Vijay S K, Vineetha Karuveettil JJ, Sravan Kumar Y, Rakesh P S, Chandrashekar J. Association of Dental Caries and Oral Health Impact Profile in 12-YearOld School Children: A Cross-Sectional Study. J Clin 
Diagnostic Res. 2018;12(9):12-5.

16. Ramirez-Puerta B, Molina-Ochoa H, Álvarez-Sánchez L. Fluorosis dental en niños de 12 y 15 años del municipio de Andes. Rev CES Odont. 2013;26(2):11-21.

17. Verdugo LS, Garcia-Rupaya CR, Del Castillo CE. Calidad de vida relacionada a la salud oral en escolares del sector urbano y rural. Cañar, Ecuador. Rev Estomatológica Hered. 2018;28(2):105-114.

18. Marcelo J, Del Castillo C, Evangelista-Alva A, Sanchez P. Calidad de vida relacionada a la salud bucal en escolares de ámbito urbano-marginal. Rev Estomatológica Hered. 2015;25(3):194-204.
19. Adunola F, Garcia I, Iafolla T, et al. Self-perceived oral health, normative need, and dental services utilization among dentate adults in the United States: National Health and Nutrition Examination Survey (NHANES) 2011-2014. J Public Health Dent. 2019; 79(1):79-90. doi: 10.1111/jphd.12300.

Recibido: 04-01-2019

Aceptado: 15-07-2019 